\title{
A Therapeutic Microparticle-Based Tumor Lysate Vaccine Reduces Spontaneous Metastases in Murine Breast Cancer
}

\author{
Brett P. Gross, ${ }^{1,2}$ Amaraporn Wongrakpanich, ${ }^{3}$ Meghan B. Francis, ${ }^{4}$ \\ Aliasger K. Salem, ${ }^{2,3}$ and Lyse A. Norian ${ }^{1,2,5,6}$
}

Received 31 July 2014; accepted 29 August 2014; published online 16 September 2014

\begin{abstract}
Metastatic breast cancer is currently incurable, and available therapies are associated with severe toxicities. Induction of protective anti-tumor immunity is a promising therapeutic approach for disseminated breast cancer, as immune responses are (i) systemic; (ii) antigen-specific; and (iii) capable of generating long-lived "memory" populations that protect against future tumor recurrences. Pursuant with this approach, we have developed a novel heterologous prime/boost vaccination regimen that reduces spontaneous lung metastases in mice with established murine 4T1 adenocarcinoma breast tumors. In our studies, mice were orthotopically challenged with luciferase-expressing 4T1 tumor cells; luciferase expression was retained in vivo, enabling us to quantitatively track metastatic tumor growth via bioluminescent imaging. On day 6 post-challenge, mice received a therapeutic "prime" consisting of bulk tumor lysates encapsulated in poly(lactic-co-glycolic) acid (PLGA) microparticles (MPs). On day 11, mice received a "boost" composed of free tumor lysates plus a cocktail of Toll-like receptor (TLR)stimulating adjuvants. Tumor progression was monitored in vaccinated and untreated mice for 25 days, a time at which $100 \%$ of untreated mice had detectable lung tumors. PLGA MPs injected subcutaneously trafficked to draining lymph nodes and were efficiently phagocytosed by dendritic cells (DCs) within $48 \mathrm{~h}$. Our combination therapy reduced metastatic lung tumor burdens by $42 \%$ and did not induce autoimmunity. These findings illustrate that vaccines based upon MP delivery of tumor lysates can form the basis of an effective treatment for metastatic breast cancer and suggest that similar approaches may be both efficacious and well-tolerated in the clinic.
\end{abstract}

KEY WORDS: breast cancer; microparticle; PLGA; tumor lysate; vaccine.

\section{INTRODUCTION}

Nearly one in eight women will develop breast cancer within her lifetime, making breast cancer a leading cause of cancer incidence and mortality worldwide (1). Localized tumors are effectively managed by surgical intervention in combination with chemotherapy, radiotherapy, hormonal therapies, or targeted biologics (2). In contrast, metastatic disease remains incurable and accounts for the majority of breast cancer-related mortalities (3). The therapeutic induction of systemic anti-tumor immunity is a promising approach

\footnotetext{
${ }^{1}$ Interdisciplinary Graduate Program in Immunology, University of Iowa, Iowa City, Iowa 52242, USA.

${ }^{2}$ Holden Comprehensive Cancer Center, University of Iowa, Iowa City, Iowa 52242, USA.

${ }^{3}$ Division of Pharmaceutics and Translational Therapeutics, College of Pharmacy, University of Iowa, Iowa City, Iowa 52242, USA.

${ }^{4}$ Department of Internal Medicine, University of Iowa, Iowa City, Iowa 52242, USA.

${ }^{5}$ Department of Urology and Fraternal Order of Eagles Diabetes Research Center, University of Iowa, Iowa City, Iowa 52242, USA.

${ }^{6}$ To whom correspondence should be addressed. (e-mail: lysenorian@uiowa.edu)
}

for treating metastatic breast cancer. Tumor-specific $\mathrm{CD} 8^{+} \mathrm{T}$ cells are of particular interest, as they are capable of: (i) systemic distribution to sites of metastasis; (ii) direct antigenrestricted cytotoxicity that limits off-target toxicity; and (iii) the formation of long-lived memory populations that can protect against future tumor relapses (4).

Cancer vaccines attempt to activate tumor-specific $\mathrm{CD} 8^{+}$ $\mathrm{T}$ cells by combining tumor-associated antigens (TAAs) with immune-stimulating adjuvants. Protective T cell responses are initiated after antigen-presenting cells, such as dendritic cells (DCs), phagocytose exogenous antigen. Importantly, DCs must also register a secondary "danger signal" to induce protective $\mathrm{T}$ cell immunity (5). This danger signal is typically an evolutionarily conserved motif present on pathogens, but it can also be triggered by functional mimics such as synthetic adjuvants (6). DCs then travel to a proximal draining lymph node (dLN), where they present antigen to naive T cells, ultimately leading to the activation of $\mathrm{CD} 8^{+} \mathrm{T}$ cells via crosspresentation of phagocytosed antigens on class I major histocompatibility complexes (7-9).

Many pre-clinical vaccine iterations exist to enhance the immunogenic cross-presentation of TAA(s). One strategy is based upon isolating DC precursors, then maturing them into 
DCs that are pulsed with TAA(s) and re-infused back into the source host, where they travel to lymph nodes and activate $\mathrm{CD}^{+} \mathrm{T}$ cells. Clinical validation of this vaccine approach occurred in 2010 with the FDA approval of sipuleucel-T (trade name Provenge $\AA$ ) for metastatic prostate cancer (10). A limitation of these interventions is the requirement for expensive and laborious ex vivo culturing and stimulation of autologous DCs. An alternative approach is to generate a vaccine protocol that is independent of ex vivo cell culture. For example, many laboratories are exploring the use of synthetic, biocompatible nanoparticles or microparticles (MPs) as vaccine delivery vehicles for encapsulated antigens [reviewed in (11)]. As prior clinical trials have illustrated that immunizing with multiple TAAs generates stronger protective immunity than immunizing with a single TAA, these studies support the use of bulk tumor lysates as an antigen source in tumor vaccines (12). Of note, encapsulation of antigens, so they are presented to the immune system in a particulate form, can enhance vaccine-induced anti-tumor immunity relative to soluble TAA $(13,14)$. For example, delivery of TAA in MPs can enhance ensuing immune responses by targeting and improving antigen uptake by DCs, thereby improving the quality and quantity of crosspresentation to naive $\mathrm{CD}^{+} \mathrm{T}$ cells (15).

In this report, we summarize a novel heterologous prime/ boost vaccination regimen that uses biodegradable, poly(lactic-co-glycolic) acid (PLGA) MPs to deliver bulk tumor lysates to the immune system. This approach significantly reduces spontaneous lung metastases that arise from established murine breast tumors, without producing autoimmune reactions or other discernable adverse events. Our results in this pre-clinical murine tumor model suggest that an optimized treatment strategy using this approach could be translated into a safe yet effective therapy for advanced breast cancer patients.

\section{MATERIALS AND METHODS}

\section{Mice}

Female BALB/c mice from 7 to 8 weeks of age were purchased from The National Cancer Institute (Frederick, MD). Upon arrival, mice were housed in specific pathogenfree conditions at the University of Iowa Animal Care Facility and were allowed to acclimate for 1 week prior to use. All murine experiments were pre-approved by the University of Iowa Animal Care and Use Committee and complied with NIH guidelines.

\section{Cells}

4T1 cells were purchased from ATCC and were cultured in RPMI-1640 medium supplemented with $100 \mathrm{IU} / \mathrm{mL}$ penicillin, $100 \mu \mathrm{g} / \mathrm{mL}$ streptomycin, $10 \mathrm{mM}$ HEPES, $2 \mathrm{mM}$ GlutaMAX ${ }^{\mathrm{TM}}$, $1 \mathrm{mM}$ sodium pyruvate (all, Life Technologies), and $10 \%(v / v)$ heat-inactivated fetal bovine serum (Thermo Scientific). Firefly luciferase-expressing 4T1 cells "4T1-fLUC" were generated using premade lentiviral particles following the manufacturer's recommended protocol (KeraFAST). Of G418 (Life Technologies), $200 \mu \mathrm{g} / \mathrm{mL}$ was used to select for in vitro luciferase retention.

\section{Tumor Challenge}

Low passage 4T1-fLUC cells were resuspended into sterile phenol red-free Hank's Balanced Salt Solution (HBSS) (Life Technologies) at a concentration of $5 \times 10^{5}$ cells $/ \mathrm{mL}$. Two hundred microliters of cells $\left(1 \times 10^{5}\right.$ cells/mouse) were injected subcutaneously into mammary gland \#9. Tumor bioluminescence was imaged 6 days later to assess consistency of tumor burdens and placement. Perpendicular caliper measurements of the largest length and width dimensions were used to calculate tumor areas.

\section{PLGA Microparticle Fabrication and Characterization}

Parental 4T1 lysates were prepared by subjecting cells to five freeze-thaw cycles accomplished by repetitive 5-min liquid nitrogen submersions followed by 5 -min water bath incubations at $37^{\circ} \mathrm{C}$.

Lysates were encapsulated into PLGA MPs (4T1 MPs) using a modified version of the double emulsion solvent evaporation method previously described (16). Of 4T1 lysates (10-20 mg/mL), $125-175 \mu \mathrm{L}$ was prepared in $1 \%$ poly(vinyl alcohol) (PVA; Mowiol® 8-88, Sigma-Aldrich) during water phase 1. Two hundred micrograms of PLGA (Resomer ${ }^{\circledR}$ RG503; Boehringer Ingelheim Pharma Gmbh \& Co.) was dissolved in $1.5 \mathrm{~mL}$ of dichloromethane (DCM, Fisher Scientific) as an oil phase. Primary emulsion was achieved by a 30 -s sonication of the water phase 1 into the oil phase using a Sonic Dismembrator Ultrasonic processor (Fisher Scientific) at $40 \%$ amplitude. This was then sonicated for $30 \mathrm{~s}$ during water phase 2 into $8 \mathrm{~mL}$ of $1 \%$ PVA to generate the secondary emulsion. The secondary emulsion was transferred into $22 \mathrm{~mL}$ of $1 \%$ PVA and stirred under a fume hood to evaporate excess DCM. MPs were then washed twice with sterile water and recovered by a 5-min centrifugation at 4,500×g using an Eppendorf centrifuge $5804 \mathrm{R}$ (Eppendorf). MPs were lyophilized using a FreeZone 4.5-L Benchtop Freeze Dry System (Labconco Corporation).

To quantify lysate loading, 4T1 MPs were incubated with $0.3 \mathrm{~N} \mathrm{NaOH}$ and, upon turning into a clear solution (approximately $45 \mathrm{~min}$ ), were neutralized with $1 \mathrm{~N} \mathrm{HCl}$. Lysate protein concentrations were then determined using the Micro $\mathrm{BCA}^{\mathrm{TM}}$ Protein Assay Kit following the manufacturer's recommended protocol (Thermo Scientific). This procedure yielded identical protein concentrations as compared to a procedure in which lysates were incubated in PBS for $45 \mathrm{~min}$ (data not shown). Six batches of 4T1 MPs were used for this report and contained an average of $4.92 \mu \mathrm{g}$ lysate/mg PLGA. 4T1 MP diameters and zeta potentials were measured using a Zetasizer Nano ZS (Malvern Instruments Ltd.) following the manufacturer's recommended protocol. 4T1 MPs had an average diameter of $2.29 \mu \mathrm{m}$ and zeta potential of $-26.83 \mathrm{mV}$. 4T1 MP morphology was evaluated using scanning electron microscopy (Hitachi S-4800, Hitachi High-Technologies).

Three distinct batches of 4T1 MPs were used to assess the release of 4T1 lysate from MPs. Thirty milligrams of 4T1 MPs were suspended in $5 \mathrm{~mL}$ of PBS, to allow for the possibility of replicate aliquots to be tested at each time point and to prevent saturation of the protein quantification assay, then incubated in a $37^{\circ} \mathrm{C}$ shaker at $300 \mathrm{rpm}$. For each time 
point, $500 \mu \mathrm{L}$ of supernatant was obtained from each tube after a 10 -min centrifugation at $4,500 \times g$. Each supernatant was used for protein quantification via the Micro $\mathrm{BCA}^{\mathrm{TM}}$ Protein Assay kit.

Rhodamine B (Sigma-Aldrich) was encapsulated in PLGA MPs as described above for the 4T1 MP protocol. One milligram of Rhodamine B was prepared in $125 \mu \mathrm{L}$ of $1 \%$ PVA for water phase 1.

\section{Vaccination Therapy}

Prime and boost therapies were administered subcutaneously into mammary gland \#4 (contralateral to primary tumor).

Prime (Day 6). 4T1 MPs were resuspended in phenol red-free HBSS and vortexed. Each mouse received a standard $25 \mu \mathrm{g}$ of $4 \mathrm{~T} 1$ lysate delivered in a $200-\mu \mathrm{L}$ volume.

Boost (Day 11). The boost was prepared (per mouse) by combining $100 \mu \mathrm{g}$ of free 4T1 lysates, $100 \mu \mathrm{g}$ of Poly(I:C) (InvivoGen) to stimulate Toll-like receptor-3 (TLR-3), 2 ng of MALP-2 (Enzo Life Sciences) to stimulate TLR-2/6, and $4 \mu \mathrm{g}$ each of CpG1585 (class A) and CpG1826 (class B) (both, Integrated DNA Technologies) to stimulate TLR-9, all aggregated in $20 \mu \mathrm{L}$ of DOTAP liposomal transfection reagent (Roche). This mixture was vortexed and incubated $15 \mathrm{~min}$ at room temperature prior to injection at $200 \mu \mathrm{L}$ per mouse in phenol red-free HBSS. The boost was administered subcutaneously to the same site as the prime.

\section{Bioluminescent Imaging}

Two milligrams per mouse of D-luciferin potassium salt (Goldbio.com) was dissolved in phenol red-free HBSS. Luciferin solution was injected intraperitoneally at $100 \mu \mathrm{L} /$ mouse, then allowed to saturate systemically for $10 \mathrm{~min}$ prior to imaging. Mice were sedated with $2.5 \mathrm{~L} / \mathrm{min}$ of oxygenated isoflurane (Piramal Healthcare Limited) and imaged using a Xenogen IVIS-200 (Caliper Life Sciences) to detect bioluminescent light emission. In vivo imaging system (IVIS) images were analyzed with Living Image v 4.2 software (Caliper Life Sciences). Bioluminescence was calculated within a standardized circular region of interest centered on primary tumors or excised lungs. To normalize IVIS results (to compensate for inter-experimental variation), individual values from therapyreceiving mice were divided by individual values from experimentally matched, therapy-free mice in a rank-based manner.

\section{6-Thioguanine Assay}

Lungs from vaccinated and untreated mice were imaged then homogenized using a gentleMACS Dissociator (Miltenyi Biotec). Lung homogenates were then digested for $15 \mathrm{~min}$ with $5 \mu \mathrm{L} / \mathrm{mL}$ of research-grade Liberase (Roche) and 37.5 $\mu \mathrm{g} / \mathrm{mL}$ of DNaseI (Sigma-Aldrich) in a $37^{\circ} \mathrm{C}$ water bath incubation with intermittent vortexing. After washing, cells were filtered $(70 \mu \mathrm{m})$ into $60.1-\mathrm{cm}^{2}$ tissue culture petri dishes
(Sigma-Aldrich). Dishes were brought to a final volume of $15 \mathrm{~mL}$ in $4 \mathrm{~T} 1$ medium and supplemented with $60 \mu \mathrm{M}$ of 6 thioguanine (Sigma-Aldrich). Plates were incubated at $37^{\circ} \mathrm{C} /$ $5 \% \mathrm{CO}_{2}$ for 9 or 14 days before colonies were fixed, stained, and quantified as previously described (17).

\section{Flow Cytometry}

Flow cytometry was performed on a Becton Dickinson LSRII cytometer and analyzed with FlowJo v 10.0.6 software (Tree Star Inc.). Rhodamine B was detected using the 585/20 filter on the 461-nm laser. APC-Cy7/CD11b (1:3,000; clone $\mathrm{M} 1 / 70)$ and APC/CD11c (1:100; clone N418) antibodies (BioLegend) were used to detect macrophages and DCs.

\section{Histology}

Lungs were inflated via tracheal insufflation using 10\% formalin and excised. Mammary glands were excised and fixed in $10 \%$ formalin. The University of Iowa Histology Research Laboratory performed tissue processing. Each organ had six $4-\mu \mathrm{m}$ sections obtained at $50-\mu \mathrm{m}$ intervals, which were mounted on slides and stained with hematoxylin and eosin (H\&E). Pathological evaluation was performed by David Meyerholz, DVM, Ph.D.

\section{Statistical Analysis}

All statistical analyses were performed using Prism v 6.0b (GraphPad Software). Data were analyzed with unpaired two-tailed Student's $t$ tests or Mann-Whitney $U$ tests as indicated in respective figure legends. Tumor areas were compared using two-way analysis of variances that included Bonferroni post hoc analyses. Linear regression was used to evaluate correlations between light flux generated via bioluminescent imaging and metastatic 4T1 lung colonies generated via the 6-thioguanine assay. Throughout the manuscript, statistical significance is indicated as $* p<0.05, * * p<0.01$, or not significant (NS).

\section{RESULTS}

\section{Evaluation of Lung Metastases in Tumor-Bearing Mice}

The majority of breast cancer mortalities result from tumor dissemination, making metastasis an important parameter to evaluate when developing breast cancer therapies (18). The murine 4T1 mammary adenocarcinoma model is ideal for pre-clinical evaluations because when orthotopically placed into mammary tissue, primary tumors spontaneously metastasize to the lungs, similar to human breast cancer $(17,19)$. In addition, 4T1 cells lack expression of estrogen receptor, progesterone receptor, and Her2/neu, making this a pre-clinical model for aggressive triple-negative breast cancer (17). To track tumor progression and metastasis, we transduced 4T1 cells to express firefly luciferase ("4T1-fLUC" cells). Using luciferase bioluminescence in 4T1-fLUCchallenged mice, we observed sporadic metastases to liver, spleens, bones, and brains of mice (not shown). However, by day 25 post-tumor challenge, metastases were reproducibly observed only in the lungs of mice (Fig. 1). IVIS background 


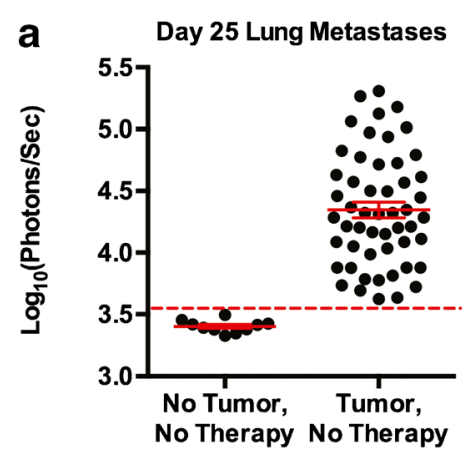

b

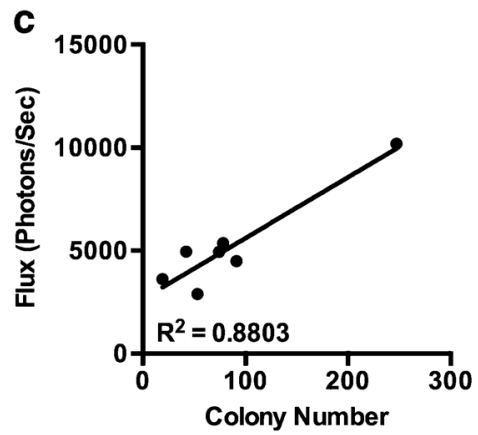

d
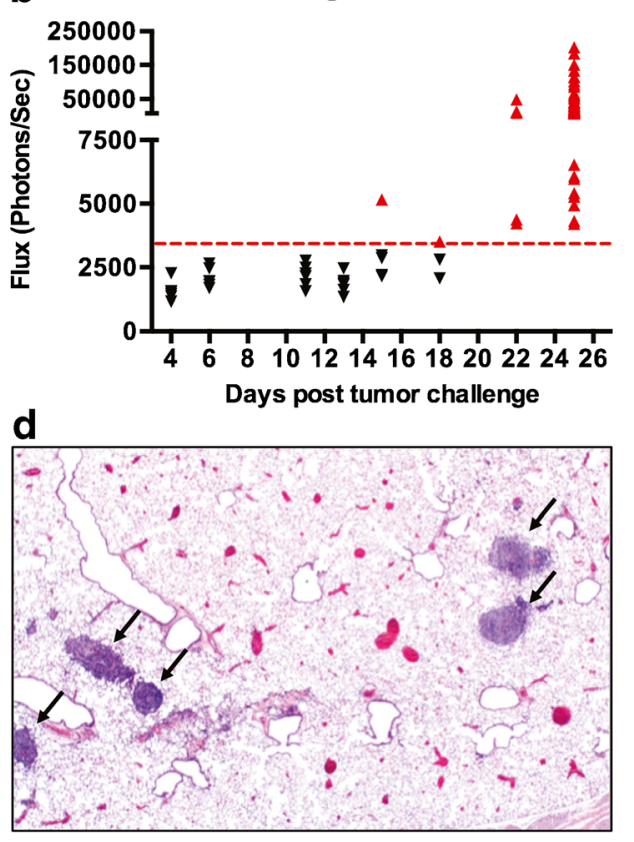

Fig. 1. Characterization of spontaneous lung metastases in the 4T1-fLUC model. a Excised lungs from ten tumor-free mice were IVIS-imaged to assess background luminescence. The metastatic incidence threshold (dashed red line) was defined as 3 S.D. above this mean. In the absence of therapy, $100 \%(n=52)$ of mice have lung metastases by day 25 after s.c. $4 \mathrm{~T} 1-$ fLUC challenge. Mean \pm S.E.M. b Excised lungs of 4T1-fLUC-challenged mice were IVISimaged to determine metastatic kinetics. Black down-pointing triangle: incidence-free mice; red up-pointing triangle: incidence-positive mice. Data are pooled from two to ten experiments using three to five mice per time point. c Excised lungs of 4T1-fLUCchallenged mice were IVIS-imaged on day 25 then homogenized and cultured for 6thioguanine colony-forming assays. Linear regression analysis indicates a positive correlation between these two independent measures of lung tumor burden. Data from one experiment, representative of two, are shown. d H\&E-stained lung sections from 4T1fLUC-bearing mice indicate the presence of multiple metastatic tumors (arrows). a-c Each data point represents bioluminescent tumor burdens from excised lungs

luminescence was determined by imaging excised lungs from ten tumor-free mice. A metastatic incidence threshold was then defined as 3 standard deviations above the mean luminescence of the tumor-free values. Using this threshold, we found that $100 \%$ of mice have IVIS-detectable lung metastases at 25 days after 4T1-fLUC challenge (Fig. 1a). We next determined the kinetics of metastasis by imaging excised lungs at predetermined time points. Metastases were first detectable by IVIS imaging on day 15 , with $100 \%$ incidence by day 22 (Fig. 1b). 4T1 cells are inherently resistant to the antimetabolite 6-thioguanine, allowing for the quantification of tumor burdens via an established colony-forming assay that is independent of luciferase expression (17). To assess whether luciferase is retained in vivo in the absence of selective pressure, we imaged excised lungs from mice with established 4T1-fLUC tumors, then subsequently performed colony-forming assays in the presence of 6-thioguanine. In two independent experiments, using a mixture of vaccinated and untreated mice, positive linear correlations were found between lung tumor burdens as measured by IVIS imaging and the colony-forming assay $\left(R^{2}=0.8803\right.$ and 0.8606 ; Fig. $1 \mathrm{c}$ and data not shown). These experiments demonstrate that luciferase is retained in vivo and provides an accurate method for quantifying tumor burdens. We subsequently used histological evaluation to validate the presence of lung metastases that were detected by bioluminescent imaging (Fig. 1d). As expected, overt 4T1 metastases were observed with H\&E staining. These results illustrate the robustness of our tumor model for use in developing a MP-based immunotherapy for metastatic breast cancer.

\section{Sustained Tumor Lysate Release from PLGA MPs}

Our vaccination strategy utilizes an initial prime that consists of tumor lysate-loaded PLGA MPs (4T1 MPs). Freeze-thawed parental 4T1 lysates were encapsulated using a double emulsion solvent evaporation method. Six batches of 4T1 MPs were utilized in these studies; the average MP had an average of $4.92 \mu \mathrm{g}$ lysates/mg of PLGA, diameter of 2.29 $\mu \mathrm{m}$, and zeta potential of $-26.83 \mathrm{mV}$. Scanning electron photomicrographs revealed that 4T1 MPs had spherical and smooth morphologies (Fig. 2a). To evaluate PLGA hydrolysis, 4T1 MPs were cultured in PBS at $37^{\circ} \mathrm{C}$, and subsequent 4T1 lysate release was assessed using Micro BCA assays. As expected, release of 4T1 lysates was sustained over time, with approximately $29 \%$ released by day 20 (Fig. 2 b). These data suggest that at a neutral $\mathrm{pH}$, the majority of non- 

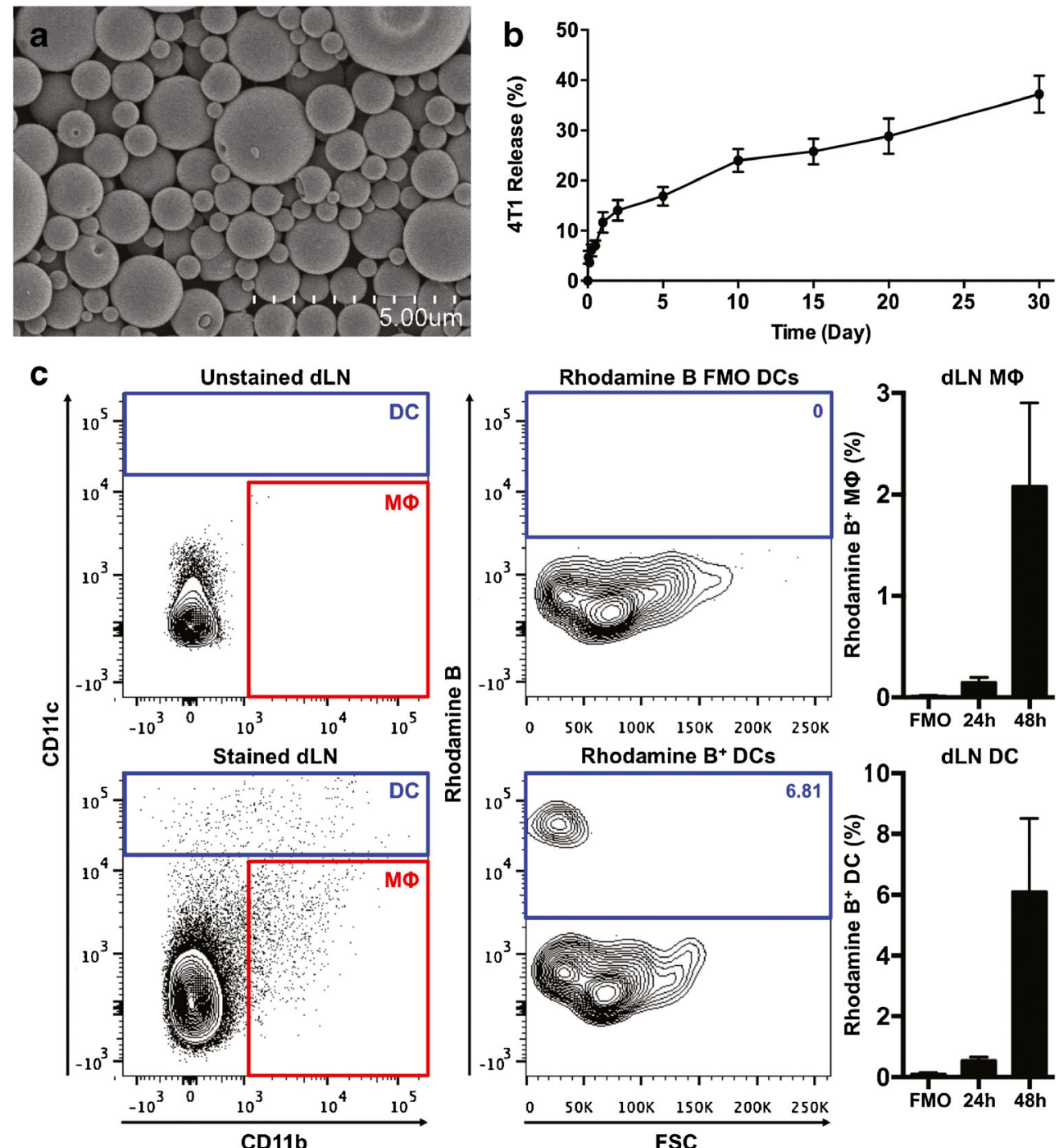

Fig. 2. PLGA MPs are efficiently phagocytosed by DCs and MФs in vivo. a Scanning electron photomicrograph of 4T1-loaded PLGA MP. b 4T1 lysate release profile from PLGA MPs cultured in PBS at $37^{\circ}$ C. Data from three batches of 4 T1 MPs. Mean \pm S.E.M. c In vivo trafficking of Rhodamine Bloaded PLGA MP. Left: Representative flow cytometry plots indicating the gating strategy for DC and МФ populations in the vaccine-dLN (inguinal). Center: Rhodamine B gating defined using FMO controls. Right: Frequency of Rhodamine B-positive phagocytes in the dLN 24 and $48 \mathrm{~h}$ after MP injection. Data shown (mean \pm S.E.M.) are pooled from two experiments using a total of eight mice/time point

phagocytosed 4T1 MPs would remain intact in vivo, allowing for efficient uptake by DCs and/or macrophages (MФs), followed by subsequent MP degradation in intracellular phagolysosomes.

\section{PLGA MPs Are Efficiently Phagocytosed In Vivo}

We next examined the efficiency with which subcutaneously administered PLGA MPs reached the vaccine-dLN, the site where naive TAA-specific $\mathrm{CD}^{+} \mathrm{T}$ cells would become activated. To assess MP phagocytosis and trafficking in vivo, we generated Rhodamine B-loaded PLGA MPs (Rhodamine MPs). Rhodamine MPs were administered subcutaneously, in the same manner as would be used for 4T1-loaded MPs in vaccinated mice. Vaccine-dLNs were isolated 24 and $48 \mathrm{~h}$ later, then analyzed by flow cytometry for Rhodamine Bpositive DCs (CD11 $\mathrm{c}^{\text {high }}$ ) and MФs (CD11b ${ }^{+}$CD $11 c^{-/ \text {int }}$ ) (Fig. 2c). Previous work had shown that MPs $>200 \mathrm{~nm}$ in diameter do not enter lymphatic ducts directly but instead require uptake by either DC or MФs that subsequently traffic to dLNs (20). To assist in defining Rhodamine B-positive events while simultaneously reducing false positives, we employed Fluorescence Minus One (FMO) controls for Rhodamine B (21). These data indicate that $0.15 \%(24 \mathrm{~h})$ and $2.08 \%(48 \mathrm{~h})$ of dLN MФ were Rhodamine B-positive, whereas $0.55 \%(24 \mathrm{~h})$ and $6.10 \%(48 \mathrm{~h})$ of DCs were Rhodamine B-positive. Thus, PLGA MPs appear to be preferentially phagocytosed by DCs that migrate to dLNs, facilitating antigen presentation to naive $\mathrm{T}$ cells. 


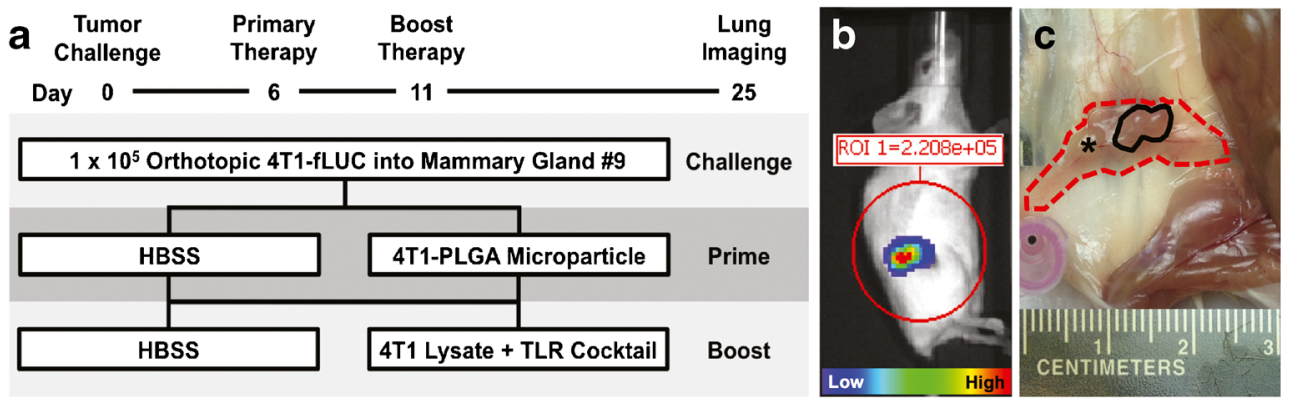

Fig. 3. Overview of heterologous prime/boost vaccination regimen. a Schematic outline of vaccine protocol. b, c Established 4T1-fLUC tumors at day 6 in the same mouse. b IVIS image of the primary tumor showing standardized region of interest. c Orthotopic placement of 4T1-fLUC tumor (black outline) within mammary gland \#9 (dashed red outline). Asterisk denotes inguinal lymph node

\section{Tumor Lysate Delivery in PLGA MPs Reduces Lung} Metastases

Immunotherapies are primarily used in the clinic to treat patients with advanced-stage cancers that have disseminated to multiple organs. We therefore wanted to evaluate the ability of a vaccination protocol based upon PLGA MP delivery of bulk tumor antigens to control both established primary tumors and metastatic tumors. Our vaccination protocol is outlined in Fig. 3a. Female BALB/c mice were orthotopically challenged with 4T1-fLUC cells, and tumors were allowed to progress for 6 days without intervention, at which time distinct primary tumors were readily detectable by palpation and IVIS imaging (Fig. 3b). On day 6, mice were given a prime of 4T1 MPs, followed 5 days later by a boost comprising free 4T1 lysates in combination with a previously described cocktail of TLR agonists (22). This rapid prime boost strategy had been shown previously to generate robust $\mathrm{CD}^{+} \mathrm{T}$ cell responses to viral challenge (23).

Vaccine efficacy was initially evaluated by comparing primary tumor burdens of vaccinated and untreated mice. We found no differences in the primary tumor areas of mice receiving the prime alone, boost alone, or full therapy (4T1 MP prime + boost), as compared to control mice that received no therapy (Fig. 4a). In contrast, lung metastases were markedly reduced by our full therapy (Fig. 4b). As demonstrated in Fig. 4c, when lung tumor burdens were quantified with bioluminescent imaging, there were significant ( $p=0.0041$, two-tailed Student's $t$ test) differences between mice that received the prime + boost therapy relative to experimentally matched, untreated mice. No significant differences in lung tumor burdens were achieved when either the prime or the boost was given alone. When each treatment group was normalized to its respective untreated control, we observed a $42 \%$ reduction in lung tumor burdens in mice that received the full therapy relative to no therapy controls (Fig. 4d). Of note, lung tumors in mice receiving the full therapy were significantly reduced compared to mice that received either the prime alone or the boost alone (Fig. 4d). Importantly, mice receiving the full therapy showed no adverse reactions to this heterologous vaccination protocol (data not shown), suggesting that a similar strategy could be translated into clinical use with minimal side effects. Thus, PLGA MP delivery of bulk tumor lysates, when used as part of an immunostimulatory prime/boost vaccination strategy, significantly reduces spontaneous lung metastases in an aggressive murine breast cancer model.

\section{Vaccination Therapy Does Not Induce Autoimmunity}

In addition to evaluating therapeutic efficacy, we assessed whether our vaccination strategy induced harmful autoimmune reactions to self-antigens that were shared by breast carcinoma cells and normal breast epithelial cells (24). To address this concern, we vaccinated tumor-free mice with our full therapy, as this would allow ample time for even delayed autoimmune reactions to develop. We harvested lungs and mammary pads from vaccinated and untreated control mice 100 days later. Organs were processed and evaluated by a pathologist for evidence of autoimmunity: inflammation, leukocytic infiltration, and loss of normal tissue architecture. Vaccinated mice displayed no increase in overt inflammation or cellular infiltration relative to untreated mice in either the lungs or vaccinated mammary pads (Fig. 5). Therefore, our vaccination protocol based upon PLGA MP delivery of bulk tumor lysates, followed by administration of free tumor lysates with multiple TLR adjuvants, can effectively reduce spontaneous lung metastases in mice without inducing autoimmunity or other noticeable adverse events.

\section{DISCUSSION}

Robust vaccine-mediated induction of anti-tumor $\mathrm{CD}^{+}$ $T$ cells holds great potential for treating metastatic cancers of the breast and other organs. Here, we demonstrate that heterologous PLGA MP vaccination with tumor lysates can lead to systemic protection against distal lung metastases. Tumor lysates contain multiple TAAs capable of inducing a broad repertoire of polyclonal $\mathrm{T}$ cell responses. Because of this, immune responses against tumor lysates can target heterogeneous tumor cell populations in a manner few other interventions achieve. Even tumor cells that do not express vaccinated TAAs can be targeted via epitope spreading, augmenting the ability of vaccine therapies to inhibit tumor progression $(25,26)$. Furthermore, vaccinating with bulk tumor lysates avoids the need to identify TAAs prior to therapy, reducing the time between tumor diagnosis and treatment. Clinically, the frontline treatment for breast cancer is surgical resection of primary mammary tumors. This means that bulk tumor lysates could be acquired during the course of 


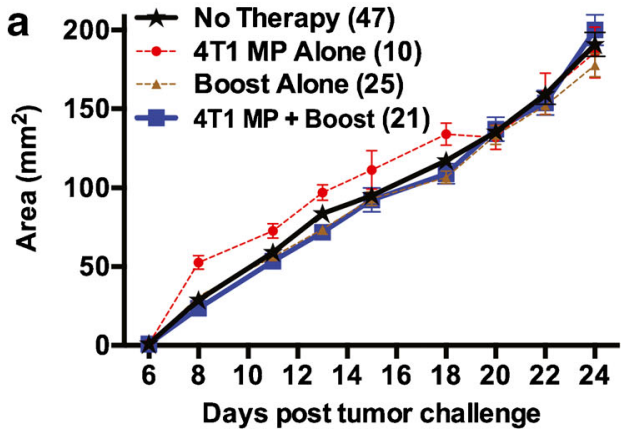

C

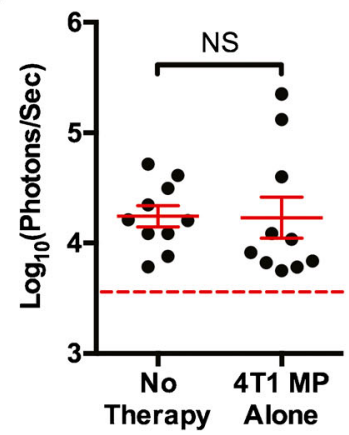

Boost Alone

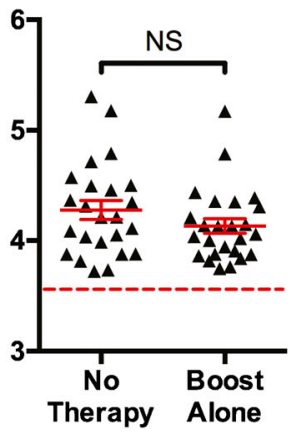

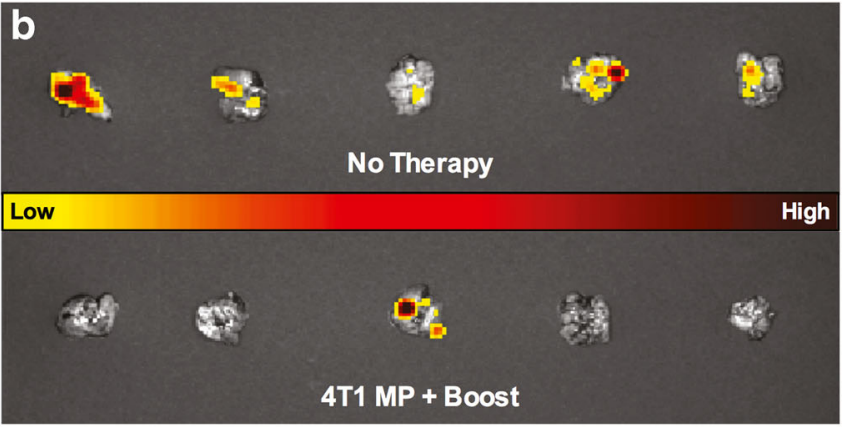

Prime + Boost

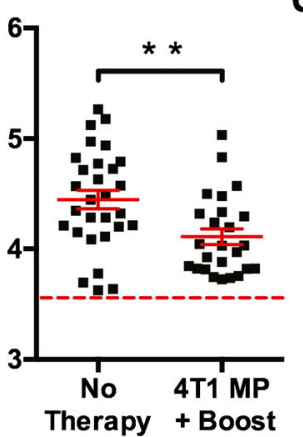

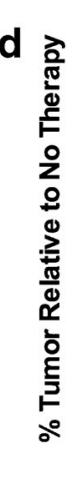

Normalized Lung Metastases

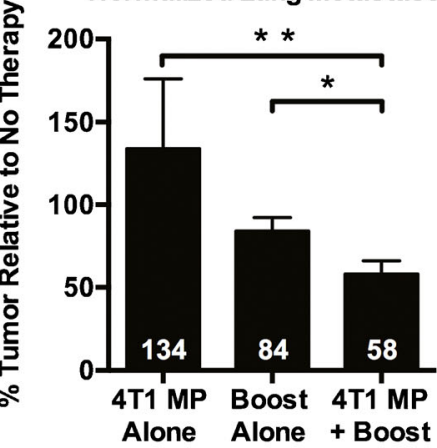

Fig. 4. Combinatorial vaccine therapy reduces spontaneous lung metastasis. a Primary tumor kinetics measured by calipers. Data from two to nine experiments using the total number of mice are indicated in parentheses. b Standardized representation of IVIS-imaged excised lungs from vaccinated and untreated 4T1-fLUC-bearing mice at day 25. c Experimentally matched lung tumor burdens from 4T1-fLUC-challenged mice that either did or did not receive therapy, as indicated. Dashed red line indicates the metastatic incidence threshold, as defined in Fig. 1a. Data points indicate tumor burdens of individual excised lungs, pooled from two to six experiments with significance evaluated by two-tailed unpaired Student's $t$ tests. d Lung metastases normalized for inter-experimental variation. Overlaid numbers indicate the mean lung tumor burdens for each treatment group relative to matched untreated controls. Groups compared using Mann-Whitney $U$ tests. a-c Mean \pm S.E.M. $N S=$ not significant; $* p<0.05 ; * * p<0.01$

standard care, incorporated into PLGA MPs, then administered as part of an adjuvant therapy, resulting in a personalized vaccine against tumor recurrence.

The in vivo delivery of tumor lysates in our vaccine was augmented by encapsulation in PLGA MPs. The PLGA shell provides a physical barrier that protects TAAs from extracellular degradation prior to phagocytosis by antigenpresenting cells. In addition, PLGA displays adjuvant properties capable of stimulating DC maturation (27,28). After phagocytosis occurs, PLGA encapsulation enhances phagosomal processing that promotes the cross-presentation of TAAs for $\mathrm{CD}^{+} \mathrm{T}$ cell activation (29). Additionally, PLGA
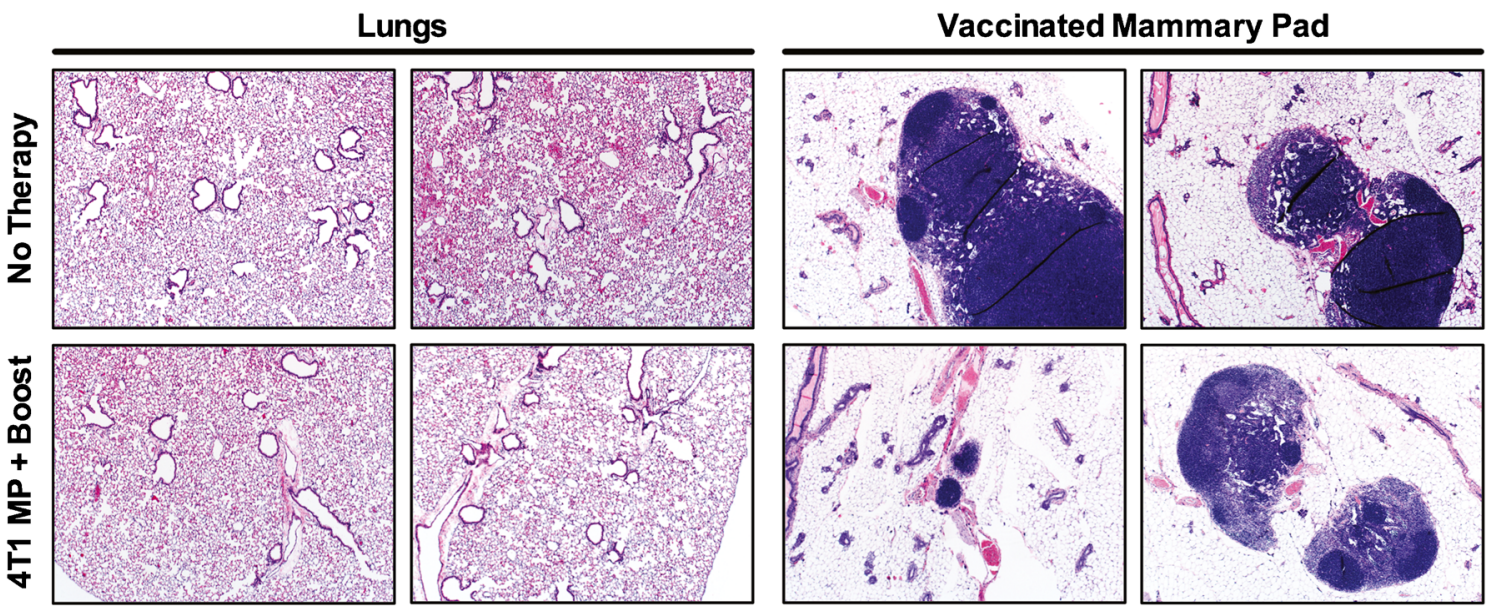

Fig. 5. Vaccine therapy does not induce autoimmunity. Tumor-free mice were fully vaccinated as summarized in Fig. 3 a. Lungs and mammary pads were collected 100 days later. H\&E-stained tissues appear normal at $\times 2$ magnification, with no evidence of autoimmunity. Representative images selected from three vaccinated and two control mice, with six distinct sections evaluated per organ 
is a highly biocompatible polymer that is approved for human use by the FDA and European Medical Agency.

After priming with 4T1 MPs, we boosted with free tumor lysates plus an adjuvant cocktail. The TLR adjuvant cocktail was adapted from Zhu et al., where this specific TLR combination enhanced the quality and magnitude of vaccine-induced $\mathrm{T}$ cell responses to HIV envelope protein (22). In our study, both the prime and boost were administered contralateral to the established tumor, to avoid immunosuppression present in the tumor-dLN (30-32). We also incorporated a relatively low inflammatory prime with highly inflammatory boost, which has been shown to enhance memory $\mathrm{CD}^{+} \mathrm{T}$ cell formation (23). Ongoing experiments in our laboratory are examining the contributions of $\mathrm{CD} 8^{+}$ versus $\mathrm{CD}^{+} \mathrm{T}$ cells to vaccine-dependent clearance of spontaneous lung metastases, and preliminary data suggest that our prime/boost strategy is inducing protective $\mathrm{CD} 8^{+} \mathrm{T}$ cell immunity (data not shown).

To improve the translational relevance of our vaccine protocol, we delayed initial vaccination until the tumor was well-established. Although our vaccine failed to reduce primary tumor growth, this was not completely unexpected due to the high tumor challenge dose administered, the rapid kinetics of 4T1 outgrowth, and the robust immunosuppression present in the primary tumor environment (33). In contrast, our full therapy was able to significantly reduce lung tumor burdens in treated mice. Because surgical resection is the standard of care for localized breast tumors, and no curative therapy currently exists for metastatic breast cancer, our treatment has clinical translatability for use in advanced cancer patients. As the use of checkpoint blockade inhibitors, such as anti-PD1 and anti-PDL1, is producing exciting responses in patients with metastatic melanoma and renal cancer, it would be of interest to determine if we could amplify the protective immune responses triggered by our therapy, via administration of checkpoint blockade inhibitors after our day 11 boost (34).

Immunotherapy for breast cancer is an increasingly attractive treatment option for patients with advanced disease. Monoclonal antibodies, such as trastuzumab and pertuzumab that target the Her2 receptor, are already in use clinically. While effective, their use is limited to a subset of patients with $\mathrm{Her} 2^{+}$tumors. Multiple ongoing clinical trials are evaluating vaccines against single or multi-peptide breast TAAs. Some vaccines are building upon the success of sipuleucel-T in prostate cancer by incorporating GM-CSF to induce DC maturation (trials \#NCT01479244 and \#NCT01660529, www.clinicaltrials.gov). Other strategies include the addition of Poly-ICLC (trial \#NCT01532960), a TLR3 adjuvant, or the ectopic expression of interleukin-12 to enhance $\mathrm{T}$ cell-mediated anti-tumor immunity (trial \#NCT00849459). Our PLGA MP therapy makes use of several of these ideas, as PLGA MPs have been shown to induce DC maturation after being phagocytosed, and our use of multiple TLR agonists in the boost protocol would further promote DC maturation and subsequent $\mathrm{T}$ cell activation. Another promising approach being developed is that of in situ immunization, which increases TAA expression via stereotactic radiation and lowers $\mathrm{T}$ cell activation thresholds via administration of an agonistic OX-40 antibody (trial
\#NCT01862900). In addition, other studies are combining low-dose chemotherapy with vaccines to selectively modulate the immune system. For example, one ongoing trial employs low-dose cyclophosphamide (trial \#NCT00978913), which has been shown to selectively deplete regulatory $\mathrm{T}$ cells, a potent mediator of pro-tumorigenic peripheral tolerance (35). These trials illustrate the intense interest in generating cell-mediated immunity against metastatic breast cancer and other tumors and underscore the potential for an efficacious and safe MPbased vaccination strategy to warrant clinical testing in advanced breast cancer patients.

Although there are considerable advantages to using tumor lysates as a source of TAAs, there is also an increased risk for triggering vaccine-induced autoimmunity against shared selfantigens (24). Fortunately, safeguards exist that limit autoreactive $\mathrm{T}$ cell activation in the form of central (developmental) and peripheral (acquired) tolerance (36). A significant challenge in therapeutic vaccine development is therefore overcoming peripheral tolerance to TAAs without inducing harmful autoimmunity to the self-antigens also found in tumor lysates. To assess autoimmunity in response to our vaccine, we treated tumor-free mice with our full therapy (4T1 MP prime + boost) and evaluated the pathology of susceptible organs 100 days later. As tumor-free mice would experience no tumor-mediated immune suppression, this approach would facilitate the progression of autoimmunity, if such a response were to develop. In addition, vaccinating tumor-free mice permitted us to extend the length of the evaluation period, to allow more time for potential autoimmune responses to develop. Despite these caveats, we found no evidence of ongoing or previous autoimmune reactions, illustrating the safety of our approach.

\section{CONCLUSIONS}

In this report, we demonstrate that therapeutic vaccination based upon PLGA MP delivery of tumor lysates is effective at reducing spontaneous metastases in a pre-clinical breast cancer model. The lack of vaccine-induced autoimmunity and the use of FDA-approved reagents highlight the translatability of this work. Tumor vaccines fill an emerging niche in comprehensive cancer care. There is recent clinical evidence to suggest that inducing polyclonal anti-tumor $\mathrm{T}$ cell responses against TAAs in heterogeneous tumor populations can efficiently inhibit disseminated tumor progression (37). The use of PLGA MPs offers many unique features for antigen delivery, as MPs can be used to co-encapsulate adjuvant or target cellular subsets via surface modifications $(11,38)$. Many proven strategies exist to further enhance the efficacy of our first-generation PLGA-tumor lysate-based vaccine, and this remains an active area of research for our laboratories. Given the surging interest in using cell-mediated immunity to protect against metastatic tumor recurrence, the tumor lysate/MP-based vaccination strategy described here has potential for further development into a clinically viable treatment option.

\section{ACKNOWLEDGMENTS}

We would like to thank our University of Iowa colleagues Vijaya B. Joshi \& Sean M. Geary (Division of Pharmaceutics and Translational Therapeutics, College of 
Pharmacy), Allison F. Christiaansen (Department of Microbiology), and Steven M. Varga (Department of Microbiology and Interdisciplinary Graduate Program in Immunology) for their technical advice and assistance with manuscript preparation. This research was supported by the University of Iowa start-up funds (LAN), the Holden Comprehensive Cancer Center Breast Cancer Research Group (LAN, AKS), NCI grant 1R01CA181088-01 (LAN, AKS), and T32 Immunology Predoctoral Training Fellowship 5T32 AI007485 (BPG). Data used in this publication were obtained using the equipment and faculty of the Central Microscopy Research Facility, Comparative Pathology Laboratory, Flow Cytometry Facility, and Histology Research Laboratory core research facilities at the University of Iowa. These core facilities are funded with support from NCI grant P30CA086862.

\section{REFERENCES}

1. DeSantis C, Ma J, Bryan L, Jemal A. Breast cancer statistics, 2013. CA: Cancer J Clin. 2014;64(1):52-62.

2. Sledge GW, Mamounas EP, Hortobagyi GN, Burstein HJ, Goodwin PJ, Wolff AC. Past, present, and future challenges in breast cancer treatment. J Clin Oncol: Off J Am Soc Clin Oncol. 2014;32(19):1979-86.

3. Gonzalez-Angulo AM, Morales-Vasquez F, Hortobagyi GN. Overview of resistance to systemic therapy in patients with breast cancer. Adv Exp Med Biol. 2007;608:1-22.

4. Appay V, Douek DC, Price DA. CD8+ T cell efficacy in vaccination and disease. Nat Med. 2008;14(6):623-8.

5. Blander JM, Medzhitov R. Toll-dependent selection of microbial antigens for presentation by dendritic cells. Nature. 2006;440(7085):808-12.

6. Akira S, Uematsu S, Takeuchi O. Pathogen recognition and innate immunity. Cell. 2006;124(4):783-801.

7. Castellino F, Huang AY, Altan-Bonnet G, Stoll S, Scheinecker C, Germain RN. Chemokines enhance immunity by guiding naive $\mathrm{CD} 8+\mathrm{T}$ cells to sites of CD4+ $\mathrm{T}$ cell-dendritic cell interaction. Nature. 2006;440(7086):890-5.

8. Lorenzi S, Mattei F, Sistigu A, Bracci L, Spadaro F, Sanchez M, et al. Type I IFNs control antigen retention and survival of CD8alpha(+) dendritic cells after uptake of tumor apoptotic cells leading to cross-priming. J Immunol. 2011;186(9):5142-50.

9. Platt AM, Randolph GJ. Dendritic cell migration through the lymphatic vasculature to lymph nodes. Adv Immunol. 2013;120:51-68.

10. Kantoff PW, Higano CS, Shore ND, Berger ER, Small EJ, Penson DF, et al. Sipuleucel-T immunotherapy for castrationresistant prostate cancer. N Engl J Med. 2010;363(5):411-22.

11. Joshi VB, Geary SM, Gross BP, Wongrakpanich A, Norian LA, Salem AK. Tumor lysate-loaded biodegradable microparticles as cancer vaccines. Expert Rev Vaccines. 2014;13(1):9-15.

12. Topalian SL, Weiner GJ, Pardoll DM. Cancer immunotherapy comes of age. J Clin Oncol: Off J Am Soc Clin Oncol. 2011;29(36):4828-36.

13. Prasad S, Cody V, Saucier-Sawyer JK, Saltzman WM, Sasaki CT, Edelson RL, et al. Polymer nanoparticles containing tumor lysates as antigen delivery vehicles for dendritic cell-based antitumor immunotherapy. Nanomed: Nanotechnol, Biol, Med. 2011;7(1):1-10.

14. Hanlon DJ, Aldo PB, Devine L, Alvero AB, Engberg AK, Edelson R, et al. Enhanced stimulation of anti-ovarian cancer CD8(+) $\mathrm{T}$ cells by dendritic cells loaded with nanoparticle encapsulated tumor antigen. Am J Reprod Immunol. 2011;65(6):597-609.

15. Rice-Ficht AC, Arenas-Gamboa AM, Kahl-McDonagh MM, Ficht TA. Polymeric particles in vaccine delivery. Curr Opin Microbiol. 2010;13(1):106-12.
16. Joshi VB, Geary SM, Salem AK. Biodegradable particles as vaccine delivery systems: size matters. AAPS J. 2013;15(1):8594.

17. Pulaski BA, Ostrand-Rosenberg S. Mouse 4T1 breast tumor model. Current protocols in immunology. Edited by John E Coligan [et al]. 2001; Chapter 20: Unit 202.

18. Weigelt B, Peterse JL, van't Veer LJ. Breast cancer metastasis: markers and models. Nat Rev Cancer. 2005;5(8):591-602.

19. Aslakson CJ, Miller FR. Selective events in the metastatic process defined by analysis of the sequential dissemination of subpopulations of a mouse mammary tumor. Cancer Res. 1992;52(6):1399-405.

20. Manolova V, Flace A, Bauer M, Schwarz K, Saudan P, Bachmann MF. Nanoparticles target distinct dendritic cell populations according to their size. Eur J Immunol. 2008;38(5):1404-13.

21. Roederer M. Spectral compensation for flow cytometry: visualization artifacts, limitations, and caveats. Cytometry. 2001;45(3):194-205.

22. Zhu Q, Egelston C, Gagnon S, Sui Y, Belyakov IM, Klinman $\mathrm{DM}$, et al. Using 3 TLR ligands as a combination adjuvant induces qualitative changes in $\mathrm{T}$ cell responses needed for antiviral protection in mice. J Clin Invest. 2010;120(2):607-16.

23. Pham NL, Pewe LL, Fleenor CJ, Langlois RA, Legge KL, Badovinac VP, et al. Exploiting cross-priming to generate protective CD8 T-cell immunity rapidly. Proc Natl Acad Sci U S A. 2010;107(27):12198-203.

24. Amos SM, Duong CP, Westwood JA, Ritchie DS, Junghans RP, Darcy PK, et al. Autoimmunity associated with immunotherapy of cancer. Blood. 2011;118(3):499-509.

25. Butterfield LH, Ribas A, Dissette VB, Amarnani SN, Vu HT, Oseguera $\mathrm{D}$, et al. Determinant spreading associated with clinical response in dendritic cell-based immunotherapy for malignant melanoma. Clin Cancer Res: Off J Am Assoc Cancer Res. 2003;9(3):998-1008.

26. Corbiere V, Chapiro J, Stroobant V, Ma W, Lurquin C, Lethe B, et al. Antigen spreading contributes to MAGE vaccinationinduced regression of melanoma metastases. Cancer Res. 2011;71(4):1253-62.

27. Sun H, Pollock KG, Brewer JM. Analysis of the role of vaccine adjuvants in modulating dendritic cell activation and antigen presentation in vitro. Vaccine. 2003;21(9-10):849-55.

28. Yoshida M, Babensee JE. Poly(lactic-co-glycolic acid) enhances maturation of human monocyte-derived dendritic cells. J Biomed Mat Res Part A. 2004;71(1):45-54.

29. Shen H, Ackerman AL, Cody V, Giodini A, Hinson ER, Cresswell $\mathrm{P}$, et al. Enhanced and prolonged cross-presentation following endosomal escape of exogenous antigens encapsulated in biodegradable nanoparticles. Immunology. 2006;117(1):7888.

30. Watanabe S, Deguchi K, Zheng R, Tamai H, Wang LX, Cohen PA, et al. Tumor-induced CD11b+Gr-1+ myeloid cells suppress T cell sensitization in tumor-draining lymph nodes. J Immunol. 2008;181(5):3291-300.

31. Fujita T, Teramoto K, Ozaki Y, Hanaoka J, Tezuka N, Itoh Y, et al. Inhibition of transforming growth factor-beta-mediated immunosuppression in tumor-draining lymph nodes augments antitumor responses by various immunologic cell types. Cancer Res. 2009;69(12):5142-50.

32. Deng L, Zhang H, Luan Y, Zhang J, Xing Q, Dong S, et al. Accumulation of foxp $3+\mathrm{T}$ regulatory cells in draining lymph nodes correlates with disease progression and immune suppression in colorectal cancer patients. Clin Cancer Res: Off J Am Assoc Cancer Res. 2010;16(16):4105-12.

33. Rosenberg SA, Sherry RM, Morton KE, Scharfman WJ, Yang JC, Topalian SL, et al. Tumor progression can occur despite the induction of very high levels of self/tumor antigen-specific CD8+ T cells in patients with melanoma. J Immunol. 2005;175(9):616976.

34. Topalian SL, Hodi FS, Brahmer JR, Gettinger SN, Smith DC, McDermott DF, et al. Safety, activity, and immune correlates of anti-PD-1 antibody in cancer. N Engl J Med. 2012;366(26):244354. 
35. Berd D, Mastrangelo MJ. Effect of low dose cyclophosphamide on the immune system of cancer patients: depletion of CD4+, 2H4+ suppressor-inducer T-cells. Cancer Res. 1988;48(6):16715.

36. Xing Y, Hogquist KA. T-cell tolerance: central and peripheral. Cold Spring Harbor perspectives in biology. 2012;4(6).
37. Neller MA, Lopez JA, Schmidt CW. Antigens for cancer immunotherapy. Semin Immunol. 2008;20(5):286-95.

38. Danhier F, Feron O, Preat V. To exploit the tumor microenvironment: passive and active tumor targeting of nanocarriers for anti-cancer drug delivery. J Control Release: Off J Control Release Soc. 2010;148(2):135-46. 